\title{
Elucidating the nanoscale architecture of amyloid aggregates using a polarized donut point spread function
}

Tianben Ding ${ }^{1}$ and Matthew Lew ${ }^{2}$

${ }^{1}$ Department of Electrical and Systems Engineering, Washington University in St. Louis, Missouri, USA, Saint Louis, Missouri, United States, ${ }^{2}$ Department of Electrical and Systems Engineering, Washington University in St. Louis, Missouri, USA, saint louis, Missouri, United States

Introduction

Protein misfolding and amyloid aggregation underlie various aging-related neurodegenerative diseases, such as Alzheimer's disease and Parkinson's disease [1]. Although amyloid aggregates share a common cross- $\beta$ sheet structural motif formed by similar amino acid sequences, aggregation intermediates formed during amyloid fibrillation are highly dynamic, transitory, and heterogeneous in terms of their structures and sizes. Interestingly, not all aggregation intermediates are equally toxic. A new imaging tool capable of visualizing the architecture of these aggregates with nanoscale resolution over long periods is required to understand how the structural dynamics of amyloid aggregation are correlated with cytotoxicity.

\section{Methods}

Transient amyloid binding (TAB) super-resolution (SR) microscopy [2] is a versatile method for studying a variety of amyloid structures without the need for covalent labeling or immunostaining. In TAB SR imaging, hydrophobic fluorescent molecules, such as Nile red (NR), spontaneously bind to the nanoscale grooves formed by stacked $\beta$ sheets within amyloid fibrils. Continuous transient binding and replenishment of dye molecules from the surrounding solution generates photon bursts for single-molecule localization over hours to days with minimal photobleaching. Here, we present a form of TAB imaging, TAB single-molecule orientation localization microscopy (SMOLM), for simultaneous measurement of positions and orientations of dye molecules on amyloid aggregates [3]. We show that the polarized donut point spread function (PSF), generated by adding a polarizing beam splitter and a vortex phase mask to a standard microscope system (Fig. 1AB), provides robust and sensitive measurements of the 3D orientations of single molecules. For dim fluorophores (510 detected photons and 2.3 background photons per pixel) near a refractive index interface, the donut PSF and a joint sparse deconvolution algorithm $[4,5]$ enables azimuthal $(\Phi)$ and polar $(\theta)$ angles as well as rotational diffusion $(\alpha, \beta)$ to be imaged with precisions of $\sigma \Phi=8^{\circ}, \sigma \theta=5^{\circ}$ and $\sigma_{\alpha, \beta}=23^{\circ}$ respectively without angular degeneracy. Furthermore, the improved orientation sensitivity introduced by the polarized donut PSF enables us to resolve anisotropic rotational diffusion ( $\alpha$ perpendicular to the coverslip vs. $\beta$ within the xy plane) of NR on amyloid aggregates (Fig. 1A inset, E and F) for the first time.

\section{Results and Discussion}

With a sampling density of $\sim 2.0$ molecule localizations per $\mathrm{nm}$ along the amyloid fibril, TAB SMOLM confirms that the main binding angles of NR on individual amyloid fibrils are mostly parallel to the long axis of the fibrils (Fig. 2CD). Our SMOLM images also show that the individual orientations of NR molecules are not always aligned with one another, even in neighboring structures. For example, NR molecules on the central "bulb" structure exhibit smaller polar angles $(\theta)$ than those on surrounding fibrillar structures. Further, although NR tends to wobble more along the polar $(\alpha)$ direction than the 
azimuthal $(\beta)$ direction in general when bound to fibrils $(\alpha>\beta$, Fig. 2EF), rotational diffusion varies significantly throughout the network. Note that increases in $\alpha$ and $\beta$ are not always correlated with one another, but NR appears to wobble more in both directions when bound to the central "bulb" structure or to intertwined fibers. These observations reveal heterogeneities in the structures of $\beta$-sheet assemblies within amyloid fibrillar networks that cannot be resolved by single-molecule localization alone.

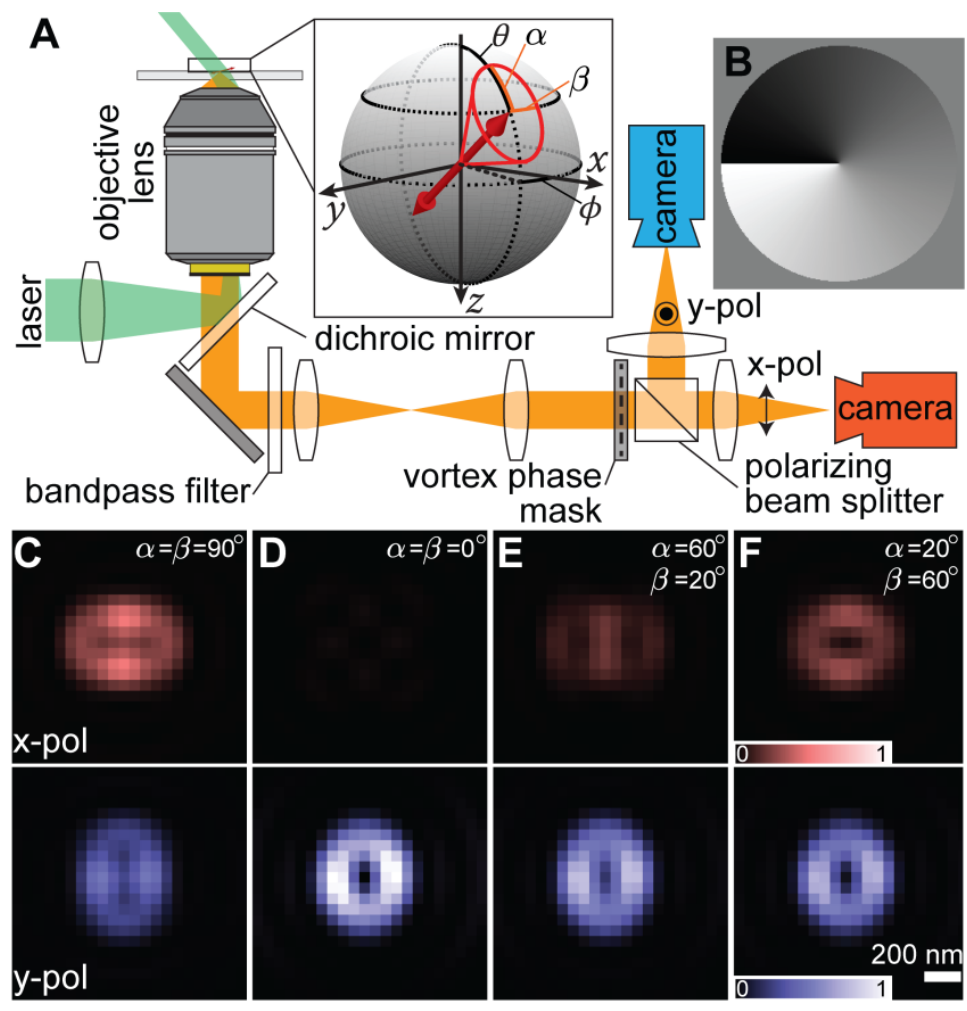

Figure 1. Fig. 1. A) Schematic of a single-molecule orientation localization microscope (SMOLM) with the polarized donut point spread function. An excitation laser is circularly polarized before being coupled into an objective for highly inclined illumination. Fluorescence from single molecules is collected by the same objective and filtered by a dichroic and a bandpass filter followed by vortex-phase-mask modulation at the Fourier plane of a $4 \mathrm{f}$ system. A polarizing beam splitter divides the modulated light into two orthogonally polarized channels before camera acquisition. Inset: The orientation of a single molecule is parameterized by an azimuthal angle $\Phi \in\left[-180^{\circ}\right.$, $\left.180^{\circ}\right)$ in the xy plane, a polar angle $\theta \in\left[0^{\circ}, 90^{\circ}\right]$ measured from the $z$ axis, and "wobbling" angles $\alpha \in\left[0^{\circ}, 90^{\circ}\right]$ (perpendicular to the coverslip), $\beta \in\left[0^{\circ}, 90^{\circ}\right]$ (within the xy plane). B) A vortex phase mask. C-F) Simulated polarized donut PSF of molecules with orientation $\left(\theta=90^{\circ}, \Phi=90^{\circ}\right)$ and various wobbling angles $(\alpha, \beta)$. 

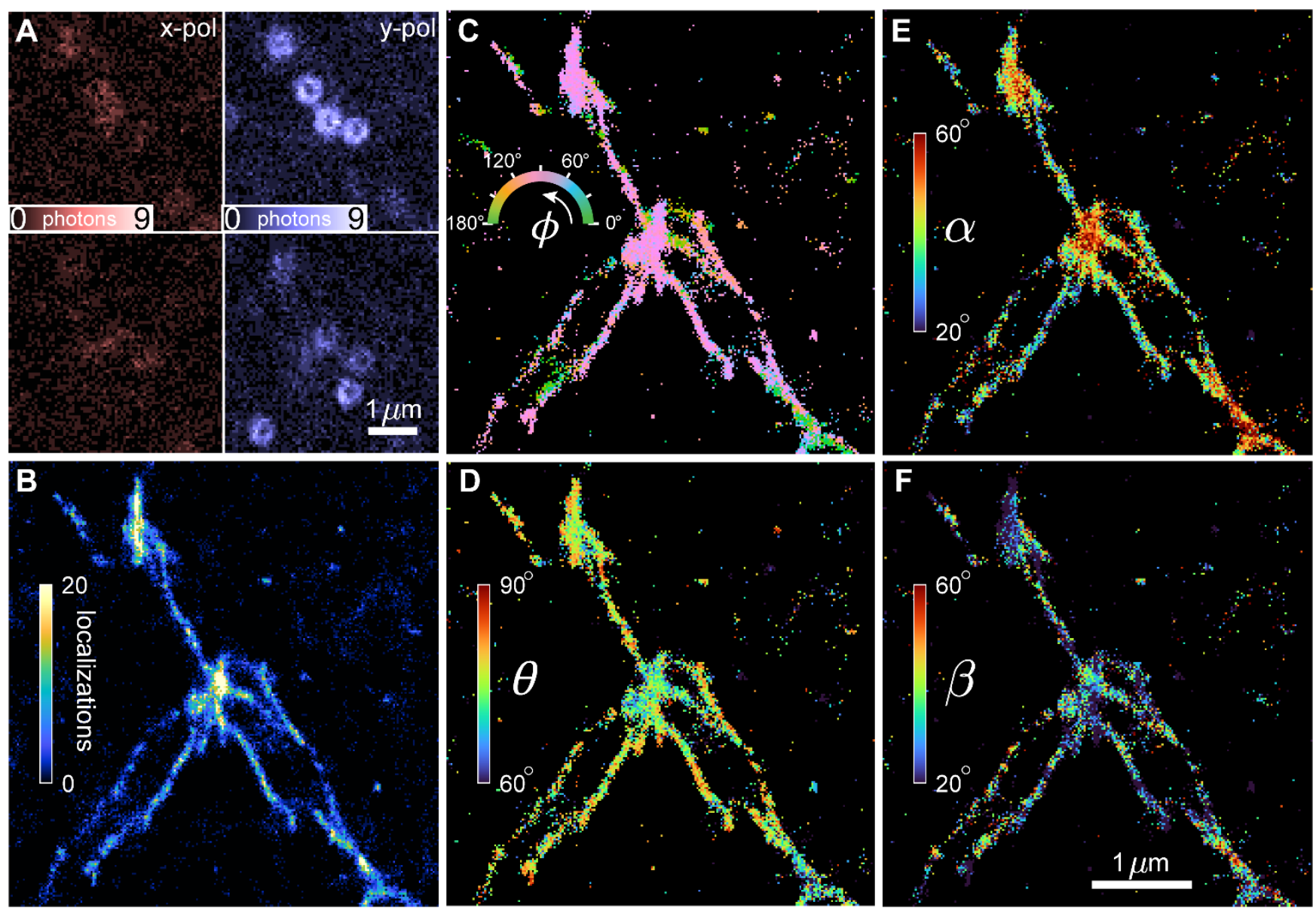

Figure 2. Fig. 2. A) Representative fluorescence raw images of single Nile red (NR) molecules on an amyloid- $\beta$ (142) fibril network. B) Single-molecule localization microscopy image of the amyloid aggregates. TAB SMOLM image, color-coded according to the mean C) azimuthal (Ф), D) polar $(\theta)$ orientations and $E, F)$ rotational diffusions $\alpha, \beta$ of NR molecules measured within each bin (20x20 nm2).

\section{References}

[1] M. G. Iadanza, M. P. Jackson, E. W. Hewitt, N. A. Ranson, and S. E. Radford, Nature Reviews Molecular Cell Biology 19, 755-773 (2018).

[2] K. Spehar, T. Ding, Y. Sun, N. Kedia, J. Lu, G. R. Nahass, M. D. Lew, and J. Bieschke, ChemBioChem 19 (18), 1944-1948 (2018).

[3] T. Ding, T. Wu, H. Mazidi, O. Zhang, and M. D. Lew, Optica 7 (6), 602-607 (2020).

[4] H. Mazidi, E. S. King, O. Zhang, A. Nehorai, and M. D. Lew, IEEE 16th International Symposium on Biomedical Imaging (ISBI) 325-329 (2019).

[5] H. Mazidi, T. Ding, and M. D. Lew, https://github.com/Lew-Lab/RoSE-O 